Disclosure of Interest: K. Zobbe: None declared, D. Prieto-Alhambra Grant/ research support from: Amgen, Servier, and UCB, Consultant for: UCB, Speakers bureau: Amgen, R. Cordtz: None declared, L. Mellemkjær: None declared, P. Højgaard: None declared, L. E. Kristensen Grant/research support from: UCB Biogen, Janssen pharmaceuticals, and Novartis, Speakers bureau: Pfizer, AbbVie, Amgen, UCB, BMS, Biogen, MSD, Novartis, Eli Lilly and Company, and Janssen pharmaceuticals, L. Dreyer: None declared

DOI: 10.1136/annrheumdis-2018-eular.2302

\section{FRI0222 ASSOCIATION BETWEEN BLOOD LEAD LEVELS AND GOUT: THE UNITED STATES NATIONAL HEALTH AND NUTRITION EXAMINATION SURVEY 2007-2014}

T.T. Cheung, M.F. Tsoi, B.M.Y. Cheung, W.C.S. Lau. Medicine, The University of Hong Kong, Hong Kong, Hong Kong

Background: Lead is a heavy metal with no physiological role in humans. It is well known that cxcessive exposure to lead is associated with cardiovascular, gastrointestinal and neurological complications. However, recent studies have demonstrated that lead can increase the tubular reabsorption of urate, resulting in hyperuricaemia and the development of gout.

Although blood lead levels are dropping in the US general population, the association between low blood lead levels ad gout remains inconclusive.

Objectives: To evaluate the relationship between serum blood lead levels and the development of gout in the US general population.

Methods: Adult participants with blood lead measurements and self reported gout in NHANES 2007-2014 were included in the analysis.

Results were analysed using SPSS complex sample module version 22. Logistic regression with sample weight adjustment was used to study the association between blood lead levels and gout. Odds ratio (OR) and 95\% confidence interval $(95 \% \mathrm{Cl})$ were estimated. Sub-group analysis was conducted in participants with blood lead level $<5 \mu \mathrm{g} / \mathrm{dL}$.

Results: 18837 adult participants were included in the analysis. 18270 participants had blood lead levels below $5 \mathrm{ug} / \mathrm{dL}$ (The current reference range for acceptable blood lead levels in healthy persons without excessive exposure to environment source of lead).

The prevalence of gout increased with blood lead levels. In the total population, the odd ratio of gout was 4.96 in subjects with blood lead levels above $2.21 \mathrm{ug} / \mathrm{dL}$. After adjusting for body mass index, hypertension, renal function and use of diuretics, the odd ratio of gout was 2.65 which remained significant.

The subgroup analysis of subjects with blood lead levels below $5 \mathrm{ug} / \mathrm{ml}$ showed similar findings. The risk of gout is increased when blood lead levels are higher than $1.43 \mathrm{ug} / \mathrm{dL}$.

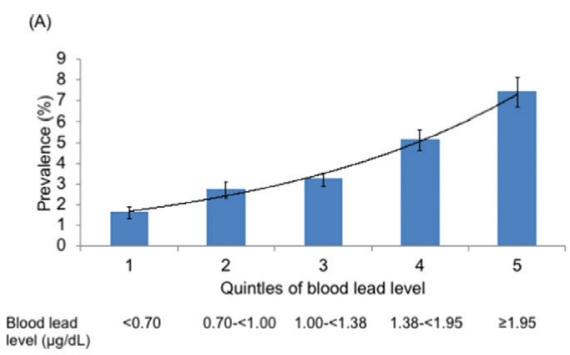

(B)

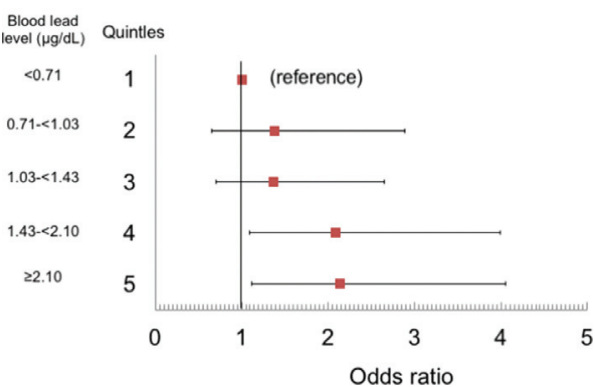

Abstract FRI0222 - Figure 1. (A) Prevalence of gout according to quintles of blood leve B) Association between quintles of blood lead level and gout at blood lead level $<5 \mu \mathrm{g} / \mathrm{dL}$
Conclusions: Low blood lead levels are associated with gout. The risk of gout is increased when the blood lead level is higher than $1.43 \mathrm{ug} / \mathrm{dL}$. Therefore, meas ures should be taken to minimise the environmental exposure to lead.

Disclosure of Interest: None declared

DOI: 10.1136/annrheumdis-2018-eular.7067

\begin{tabular}{|l|l}
\hline FRI0223 & IS PERFORMANCE OF THE 2015 ACR/EULAR GOUT \\
& CLASSIFICATION CRITERIA AFFECTED BY DISEASE \\
& DURATION AND GENDER? RESULTS FROM AN \\
& ITALIAN MULTICENTRE STUDY FOCUSED ON THE \\
& MANAGEMENT OF CRYSTAL-INDUCED ARTHRITIDES \\
& (ATTACK STUDY)
\end{tabular}

N. Ughi ${ }^{1}$, A. Zanetti ${ }^{1}$, P. Frallonardo ${ }^{2}$, A. Hoxha ${ }^{3}$, M. Lorenzin ${ }^{3}$, A. Ariani ${ }^{4}$ F. Ceccarelli ${ }^{5}$, M.A. Cimmino ${ }^{6}$, C. Scirocco ${ }^{5}$, B. Raffeiner ${ }^{7}$, A. Bortoluzzi ${ }^{8}$, A. Di Matteo $^{9}$, F. Furini ${ }^{8}$, M. Manara ${ }^{1}$, F. Salaffi ${ }^{9}$, G. Carrara ${ }^{1}$, C.A. Scirè ${ }^{1}$, R. Ramonda ${ }^{3}$. ${ }^{1}$ Epidemiology Unit, Italian Society for Rheumatology, Milan; ${ }^{2}$ Universita' degli Studi di Padova; ${ }^{3}$ Università degli Studi di Padova, Padova; ${ }^{4}$ AOU di Parma, Parma; ${ }^{5}$ Sapienza Università di Roma, Roma; ${ }^{6}$ Università di Genova, Genova; ${ }^{7}$ Ospedale di Bolzano, Bolzano; ${ }^{8}$ AOU Sant'Anna, Ferrara; ${ }^{9}$ Università Politecnica delle Marche, Ancona, Italy

Background: Gout is a common form of arthritis, but early disease and phenotypes related to gender ${ }^{1}$ may favour diagnosis misclassification, unless monosodium urate (MSU) crystals are searched for in synovial fluid (SF). The 2015 ACR EULAR gout classification criteria ${ }^{2}$ were validated in Rheumatology and primary settings, ${ }^{3}$ and the impact of disease features on performance were observed, ${ }^{4}$ yet little studied.

Objectives: To assess the performance of the 2015 ACR/EULAR gout criteria in a cohort of crystal-induced arthritides stratified by disease duration and gender in a real-life setting in Italy.

Methods: In/outpatients referring to Rheumatology Units for acute arthritis were enrolled into an ongoing multicentre cohort study designed for achieving improvement in the management of crystal-induced arthritis (ATTACk) by the Italian Society for Rheumatology. Gout was defined as MSU +SF (gold standard), irrespective of the clinical diagnosis, and the ACR/EULAR criteria were applied as full (all domains) and clinical-only set excluding imaging and SF analysis. To clas sify a patient as having clinical gout, the sufficient criterion (MSU +SF) was ignored and cutoff score $>8$ was used. Overall sensitivity and specificity were calculated. Disease duration (early if $<2$ years) was considered and gender effect explored.

Results: Of 199 adult patients (65 \pm 12 years, $75 \%$ male, 96\% Caucasian) recruited in 11 hospitals, complete SF analysis and ACR/EULAR score were obtained in 105 (53\%). MSU+were 62/105 (59\%), 47/62 (76\%) were nontophaceous and $25(40 \%)$ early diseases. Calcium pyrophosphate (CPP) was the most prevalent crystal in non-MSU+. Details on performance $(95 \%$ confidence intervals) are shown in table and on a receiver operating characteristic plot (figure 1).

Abstract FRI0223 - Table 1

\begin{tabular}{lcccc}
\hline & \multicolumn{2}{c}{ Sensitivity\% } & \multicolumn{2}{c}{ Specificity\% } \\
\hline & Full & Clinical-only & Full & Clinical-only \\
\hline Overall $\mathrm{n}=105$ & $76(63-86)$ & $71(58-82)$ & $95(86-99)$ & $81(67-92)$ \\
$<2$ years $\mathrm{n}=49$ & $48(28-69)$ & $40(21-61)$ & $100(86-100)$ & $92(73-99)$ \\
$\geq 2$ years $\mathrm{n}=56$ & $95(82-99)$ & $92(78-98)$ & $89(67-99)$ & $68(44-87)$ \\
Male $\mathrm{n}=74$ & $77(64-87)$ & $74(60-85)$ & $94(71-100)$ & $65(38-86)$ \\
Female $\mathrm{n}=31$ & $60(15-95)$ & $40(5-85)$ & $96(80-100)$ & $92(75-99)$ \\
\hline
\end{tabular}

Overall good performance and high specificity of full and clinical-only sets were observed. In early disease criteria had high specificity, but low sensitivity, while performance was stable in longstanding gout. Specificity (clinical-only) and sensitivity were low when criteria were applied to males and females, respectively. 


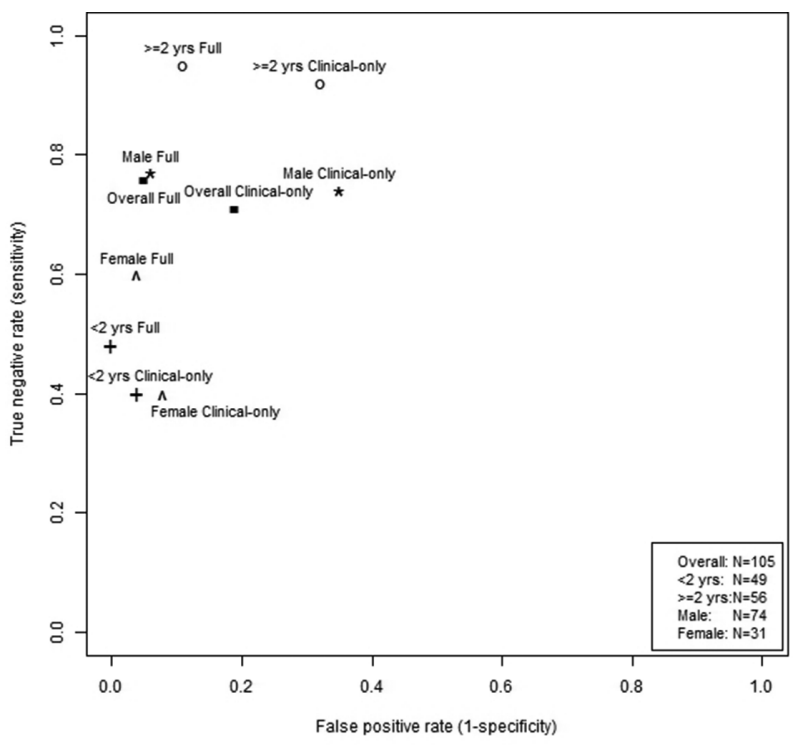

Conclusions: The overall performance of the 2015 ACR/EULAR gout criteria was good, particularly for the purpose of enrolling into trials. The impact of disease duration was confirmed ${ }^{4}$ and should be considered for misclassification issues as well as in clinical practice. Exploratory findings on gender effect seem relevant and deserve further studies.

\section{REFERENCES:}

[1] Jansen Dirken-Heukensfeldt KJM, et al. 2010.

[2] Neogi T, et al. 2015.

[3] Janssens HJEM, et al. 2017.

[4] Louthrenoo W, et al. 2017.

Disclosure of Interest: N. Ughi: None declared, A. Zanetti: None declared, P. Frallonardo: None declared, A. Hoxha: None declared, M. Lorenzin: None declared, A. Ariani: None declared, F. Ceccarelli: None declared, M. A. Cimmino Grant/research support from: Menarini, Speakers bureau: Menarini, C. Scirocco: None declared, B. Raffeiner: None declared, A. Bortoluzzi: None declared, A. Di Matteo: None declared, F. Furini: None declared, M. Manara: None declared, F. Salaffi: None declared, G. Carrara: None declared, C. A. Scirè: None declared, R. Ramonda: None declared

DOI: 10.1136/annrheumdis-2018-eular.2961

\section{FRI0224 MANAGEMENT TO REDUCING LIVER FUNCTION IMPAIRMENT WHEN INITIATING FEBUXOSTAT FOR GOUT BASED ON THE PREDICTION NOMOGRAM}

\section{J. Yu. Rheumatology, Distinct Health Care, Shenzhen, China}

Background: Febuxostat is now used widely as urate-lowering therapy in gout, The most common adverse effects of which is liver function impairment (LFI). However, it is still not known in what conditions will the LFI happen.

Objectives: To identify the potential factors associated with LFI and establish an effective prediction nomogram, and also to determine whether febuxostat with stepwise dose increase useful in reducing LFI in patients with high risk calculated basing the nomogram.

Methods: Part A: A retrospective study was performed among patients with gout. Nomogram was established based on logistic regression. The internal validation was performed via the area under the receiver operating characteristic (ROC) curve (AUC). Part B: In the follow-up prospective study, patients were divided into high-risk (>30\%) group (stepwise dose increase of febuxostat from 10 to $40 \mathrm{mg} /$ day) and low-risk ( $\leq 30 \%$ ) group (fixed-dose febuxostat $40 \mathrm{mg} /$ day) calculated basing the nomogram. Incidence rate of LFI were analysed.

Results: Part A: 306 subjects were recruited. LFI happened in 38 subjects after initiating fixed-dose febuxostat $40 \mathrm{mg} /$ day. The logistic regression multivariate analysis indicated that age, use of alcohol, chronic renal insufficiency (CRI), medication use of statin and fatty liver were significantly associated with LFI. The AUC was 0.8424 (95\% Cl: $0.765-0.920)$. Part B: 108 subjects were recruited. LFI happened in $3 / 46$ in high-risk group and 4/62 in low-risk group. The incidence rate of LFI after management basing prediction nomogram $(7 / 108,6.48 \%)$ was lower than previous study $(38 / 306,12.42 \%)$, with statistically difference $(P<0.05)$.
Abstract FRI0224 - Table 1. Multivariate logistic regression analysis of variables $(P<0.05)$ predicting LFI.

\begin{tabular}{lcc}
\hline Variable & P value & Adjusted OR (95\% Cl) \\
\hline Age & 0.004 & $1.062(1.020-1.106)$ \\
Gender & 0.507 & $0.569(0.109-2.996)$ \\
Chronic renal insufficiency (\%) & 0.038 & $5.905(1.100-31.701)$ \\
Hypertension (\%) & 0.359 & $0.573(0.174-1.884)$ \\
Coronary artery disease (\%) & 0.906 & $0.907(0.181-4.552)$ \\
Hyperlipidemia (\%) & 0.638 & $1.395(0.349-5.573)$ \\
Statin use (\%) & 0.021 & $6.608(1.324-32.970)$ \\
Fatty liver (\%) & 0.024 & $6.005(1.268-28.438)$ \\
Alcohol use (\%) & 0.028 & $2.882(1.119-7.420)$ \\
\hline
\end{tabular}

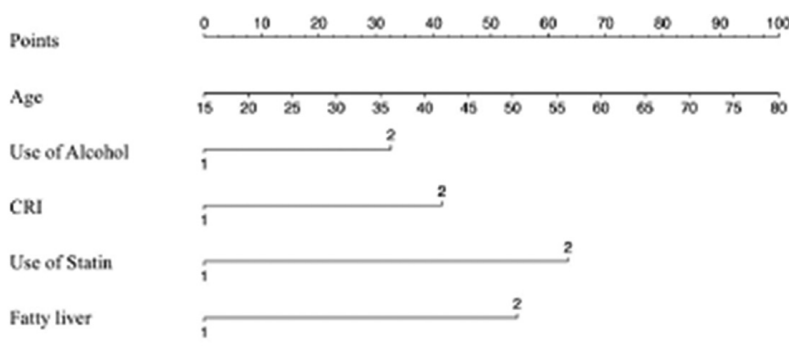

$\begin{array}{lllllllllllllll}\text { Total Points } & 0 & 20 & 40 & 60 & 90 & 100 & 120 & 140 & 100 & 180 & 200 & 220 & 240 & 200\end{array}$

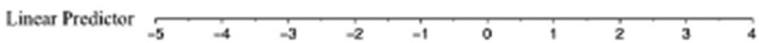

Risk of liver function abnormalities

0.102030 .40506060 .809

The nomogram allows the user to obtain the probability of liver function impairment risk when initiating febuxostat. As an example, locate the patient's age and draw a line straight upward to the "Points" axis to determine the score associated with that age. Repeat the process for each variable, and sum the scores achieved for each covariate, and locate this sum on the "Total Points" axis. Draw a line straight down to determine the likelihood of liver function impairment. CRI - chronic renal insufficiency.

Abstract FRI0224 - Figure 1. Prediction nomogram for liver function impairment in gout treated with febuxostat.

The nomogram allows the user to obtain the probability of liver function impair ment risk when initiating febuxostat. As an example, locate the patient's age and draw a line straight upward to the "Points" axis to determine the score associated with that age. Repeat the process for each variable, and sum the scores achieved for each covariate, and locate this sum on the "Total Points" axis. Draw a line straight down to determine the likelihood of liver function impairment. CRI chronic renal insufficiency.

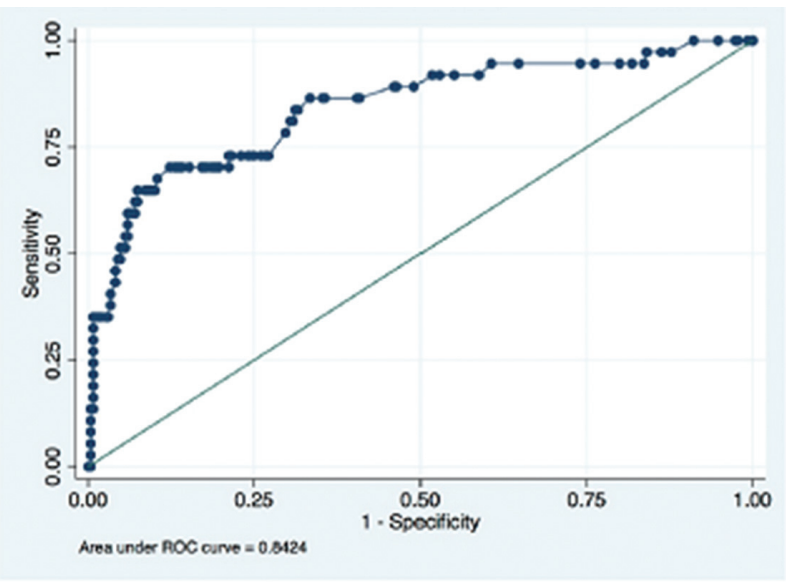

Internal validation using the receiver operating characteristic (ROC) curve. The area under the ROC curve was $0.8424,95 \%$ confidence interval $195 \% \mathrm{Cl}$ : $0.765-0.920)$

Abstract FRI0224 - Figure 2. Validation of the nomogram.

Internal validation using the receiver operating characteristic (ROC) curve. The area under the ROC curve was $0.8424,95 \%$ confidence interval $(95 \% \mathrm{Cl}$ : $0.765-$ 0.920). 